\title{
Dark malevolent traits and everyday perceived stress
}

\author{
Petri J. Kajonius ${ }^{1,2,3} \cdot$ Therese Björkman $^{2}$
}

Published online: 13 August 2018

(C) The Author(s) 2018

\begin{abstract}
Stress is a factor that greatly impacts our lives. Previous research has examined individual differences in relation to stress. However, research regarding malevolent personality traits in relation to how stress is perceived is limited. The purpose of the present study was to investigate relationships between dark malevolent personality traits; psychopathy (EPA), Machiavellianism (MACH-IV), vulnerable narcissism (HSNS), grandiose narcissism (NPI-13), and perceived stress (PSS-10) in a community sample $(N=346)$. The results showed a strong positive relationship between vulnerable narcissism and perceived stress, while grandiose narcissism and psychopathy showed a small negative relationship with perceived stress. The discussion centers on that narcissism should be treated as two separate traits, and that psychopathy and Machiavellianism overlap in relation to the experience of stress in everyday life.
\end{abstract}

Keywords Dark triad $\cdot$ Stress $\cdot$ Personality

A Narcissist, a Psychopath and a Machiavellian Walk into a bar-

The bartender asks- "who has the darkest personality out of you three?'

The Narcissist says- 'me'. The Psychopath says- 'I don't care' and the Machiavellian says- 'it's whoever I want it to be'.

Stress may be defined as the perception of being under everyday life pressures, and can be considered one of the greatest enemies of both individuals as well as organizations. Stress affects well-being, health, and ultimately performance (Cohen et al. 1993; Richardson et al. 2012). Personality psychology in particular takes interest in how stress is perceived differently by individuals. Much research focuses on traits, and particularly the Five-Factor Model (FFM; extraversion, agreeableness, conscientiousness, neuroticism, and openness; Costa and McCrae 1985). Other traits, such as dark malevolent traits, have

Petri J. Kajonius

petri.kajonius@hv.se

1 Department of Psychology, University of Gothenburg, P.O. Box 500, SE 40530 Göteborg, Sweden

2 Department of Social and Behavioral Studies, University West, Trollhättan, Sweden

3 Department of Cognitive Neuroscience, University of Skövde, Skövde, Sweden still only began to be explored (see). The most popular conceptualization of malevolent traits in the last few years has been the Dark Triad, which is constituted by subclinical psychopathy, Machiavellianism, and subclinical narcissism (Jones and Paulhus 2011). The term subclinical indicates that these traits exist in the regular population. By studying personality traits we can gain better understanding of personal motivational drives (See Jonason and Jackson 2016), and how individuals perceive their everyday pressures in everyday life (i.e., the current study's definition of stress). Malevolent traits such as the Dark Triad are known for antagonism in social situations, which could both increase or decrease perceived stress (Miller et al. 2016). Much research has linked the Dark Triad to other traits, such as Neuroticism (i.e., low anxiety), which should imply a lesser susceptibility to overall stress (Klimstra et al. 2014; Miller et al. 2016). However, arguably, a trait such as Neuroticism may differ somewhat from descriptions of everyday-stress, which may be more value-infused. The present study is one of the first to investigate relationships with malevolent personality traits using a perceived everyday life stress-scale.

\section{Stress}

The psychological concept of stress has often been unclear and only broadly defined (Lazarus 1993). Perceived stress may be defined as an individual's reaction to external problems and pressures. In the present study we consider stress as 
the perception of overall pressures in everyday life. Based on this, differences in personality traits such as the Dark Triad of malevolent traits are relevant to comprehend. What constitutes stress differs depending on trait disposition, and individual differences should be studied extensively - One perspective is that stress-related ill health is one of the most common causes of long-term sickness and decreased performance both in everyday private and professional life (Bolger and Zuckerman 1995; Cohen et al. 1983). The trait Neuroticism predicts stress the best (Miller et al. 2011). For instance, based on more than 3400 participants, Neuroticism and stress correlated highly in a Norwegian population $(r=.65$; Ebstrup et al. 2011). Earlier research has not investigated how dark traits relate to the view on everyday stress, which may be a sometimes overlooked factor relating to a host of various life outcomes (Birkás et al. 2016a; Jones and Paulhus 2010; Miller et al. 2016; Noser et al. 2014; Vazire and Funder 2006).

\section{Dark Triad}

The Dark Triad features (psychopathy, Machiavellianism, and narcissism) are usually described as overall malevolent (Paulhus and Williams 2002). Characteristic and unifying in all the Dark Triad traits is the antagonistic tendency - For example, lack of empathy, inclination to manipulate, and high self-regard (Furnham et al. 2014; Paulhus and Williams 2002; Persson et al. 2017a). Interestingly, shared variance between particularly psychopathy and Machiavellianism is often high, marked by social disinhibition, while narcissism sometimes is set apart by social skills (Lee and Ashton 2005; Paulhus and Williams 2002; Persson et al. 2017b). Previous research has found small negative connections with stress-related traits such as neuroticism (Jones and Paulhus 2010; Jones and Weiser 2014; Paulhus and Williams 2002). Former studies also suggest that narcissism should be considered in two forms (grandiose and vulnerable), and that Neuroticism may be a key in differing between the two (Miller et al. 2011; Pincus et al. 2009). The importance of studying dark personalities as well as normal personalities is that traits may be considered a foundation to how people generally view everyday life (Jones and Paulhus 2013).

Psychopathy The first malevolent trait in the Dark Triad structure coincides with particular reckless behaviors, such as aggression and impulsivity (Jones and Paulhus 2011; Noser et al. 2014). Psychopathy sometimes is considered the core of the Dark Triad, seeing how exploitation is the key component (Kajonius et al. 2016). Individuals with a high level of psychopathy are often very callous towards social norms. This could be interpreted as low perceived stress in social settings (Hare 1985; Jones and Paulhus 2013; Miller et al. 2016). This disposition aligns with research showing that psychopathy coincides negatively with stress-related traits such as Neuroticism (Birkás et al. 2016b; Gore and Widiger 2016). A concluding but tentative assumption would therefore be that the experience of stress in individuals with high levels of psychopathy is relatively low.

Machiavellianism The second malevolent trait in the Dark Triad differs from other personality features in that it is not based on any traditionally diagnosed disorder (Paulhus and Williams 2002). The Machiavellian individual is particularly characterized by manipulation, and pessimistic cynicism (Jones and Paulhus 2013; Miller et al. 2016; Paulhus and Williams 2002). Stress behaviors are less clear for Machiavellianism. Studies show no clear-cut established connection with Neuroticism (Jones and Paulhus 2010; Jones and Weiser 2014). Also, Neria et al. (2016) showed that Machiavellianism does not relate unambiguously to avoidance-motivation (a type of stress based on the Behavioral Inhibition System; BIS). They found no initial zero-order correlation, but a positive relation emerged when controlling for the other dark traits. A tentative suggestion is that Machiavellianism, since sharing much variance particularly to psychopathy, correlates negatively with perceived stress.

Narcissism The third malevolent trait is particularly characterized by feelings of grandiosity (John and Robins 1994; Raskin et al. 1991). Often narcissism can be seen in two forms; the overt form of narcissism, grandiose, and the covert form, vulnerable (Miller et al. 2010; Wink 1991). Grandiose narcissism is characterized by high self-esteem and entitlement (Gore and Widiger 2016). Vulnerable narcissism has similar features, but is additionally characterized by neuroticism, such as anxiety and low self-esteem (Gore and Widiger 2016; Miller et al. 2011). In terms of motivation, grandiose narcissism is driven by more approach-motivation, while the vulnerable type more by avoidance-motivation (Foster and Trimm 2008). Studies report varying relationships between the two forms of narcissism suggesting the trait Neuroticism (the disposition for anxiety) as the key dividing factor (Miller et al. 2011). Potential differences in perceived stress between the two types of narcissism will be relevant for the discussion on whether to continue considering these as a common disorder.

\section{The Present Study}

The general aim of the present study was to investigate relationships between malevolent personality traits (psychopathy, Machiavellianism, grandiose, and vulnerable narcissism) and perceived stress. This topic is relevant for furthering the understanding on how stress is linked to individual differences, specifically in malevolent personality traits. The specific objectives of the study were to 1 ) test whether psychopathy and 
Machiavellianism have similar relationships to perceived stress, and 2) whether the two types of narcissism show different relationships to perceived stress. Our hope is that this study may contribute to a deeper understanding of the malevolent personality traits that make up the Dark Triad.

\section{Method}

\section{Sample and Procedure}

The sample $(N=346)$ consisted of $85 \%$ women and $15 \%$ men. The age $(M=42, S D=10)$ ranged between 19 and 62 years old. $90 \%$ had a working job, and $70 \%$ reported to have attended university.

Participants were recruited by word of mouth mainly through social media to enter an online survey about stress. The questionnaire was initiated with a letter of formal notice that there were no right or wrong answers and they were asked to respond to the option that felt most correct. No reimbursement was awarded. None of the participants had to be excluded due to erratic or missing responses.

\section{Instruments}

Perceived Stress Scale (PSS-10) PSS-10 is a ten iteminstrument designed to measure present, not long-term, perceived stress. The scale taps into, for example, negative external influences at work, or present stressful life events (Cohen et al. 1993). One example question is "How often have you felt stressed in the last month?". The questions were answered on a five-digit scale from 1 (does not fit at all) to 5 (fits very well). The internal consistency was high $(\alpha=.89)$.

Elemental Psychopathy Assessment (EPA) We used the Super Short Form of EPA (EPA-SSF; Collison et al. 2016), which by 18 items measure levels of psychopathy in community populations only. One example question was "I like doing things that are risky or dangerous". The questions were answered on a five-digit scale from 1 (does not fit at all) to 5 (fits very well). This brief measurement has been found to have sufficient validity (Collison et al. 2016). Internal consistency was low $(\alpha=.58)$.

Machiavellianism (MACH-IV) MACH-IV is one of the most widely used instruments for measuring Machiavellianism (Christie and Geis 1970; McHoskey et al. 1998). One example question from the 20 item-questionnaire was "Most people are good at heart". The questions were answered on a five-digit scale from 1 (does not fit at all) to 5 (fits very well). Internal consistency was good $(\alpha=.75)$.
Grandiose Narcissism (NPI-13) The Narcisstistic Personality Inventory (NPI-13) measures the level of grandiose narcissism in community populations (Gentile et al. 2013). One example question was "I become embarrassed when people complement me" (reversed). NPI-13 consists of 13 statements that are answered by selecting one of the two specified options (A, B). Internal consistency was low $(\alpha=.56)$.

Vulnerable Narcissism (HSNS) The Hypersensitive Narcissism Scale (HSNS) with 10 items is used to assess levels of vulnerable narcissism (Hendin and Cheek 1997). One example question was "I easily get lost in my own interests, and forget about others". The questions were answered on a five-digit scale from 1 (does not fit at all) to 5 (fits very well). Internal consistency was good $(\alpha=.78)$.

\section{Statistical Analyses}

All data was analyzed in SPSS v.22, using zero-order correlations and regression models. The following recommendations based on Gignac and Szodorai (2016) were used: $r<.10$ is a trivial unrelated relationship, $r<.20$ (weak), $r<.30$ (medium), while above $r=.30$ a strong relationship. Due to sample size and consequent confidence intervals these limits should not be considered as cut-offs, but only as tentative guidelines. Normal distribution was checked and all scales were within limits.

\section{Results}

The overall aim of the present study was to investigate the relationships between malevolent personality traits and perceived stress. Table 1 summarizes the study variables. Psychopathy, Machiavellianism, and grandiose Narcissism had weak to trivial correlations with perceived stress, while vulnerable narcissism showed a strong correlation with perceived stress.

The first objective was to analyze how psychopathy and Machiavellianism compared in relation to perceived stress. Based on research on the structure of the Dark Triad, it has been suggested that these are similar almost to the point of inseparable. When entering only these two traits in a regression model

Table 1 Correlations between study variables

\begin{tabular}{llllll}
\hline Instrument & 1 & 2 & 3 & 4 & 5 \\
\hline 1 Perceived stress & & & & \\
2 Vulnerable narcissism & $.44^{* *}$ & & & \\
3 Grandiose narcissism & $-.14^{* *}$ & .04 & & \\
4 Psychopathy & $-.15^{* *}$ & .09 & $.42^{* *}$ & \\
5 Machiavellianism & .08 & $.31^{* *}$ & $.26^{* *}$ & $.38^{* *}$ \\
\hline
\end{tabular}

$* * p<.01 . N=346$ 
$(F(2,344)=8.90, p<.001)$ with perceived stress as the dependent variable, psychopathy showed a weak negative relationship ( $\beta=-.22, p<.001)$, and Machiavellianism a weak positive relationship $(\beta=.17, p<.01)$. Notably, both traits showed increased relationships to perceived stress compared to the zeroorder correlations (Table 1), suggesting that some of the errorvariance was absorbed. The two traits also showed sizable relationship to each other $(r=.38)$.

The second objective was to analyze whether the two forms of narcissism had different relationships to stress. When entering only these two narcissistic traits in a regression model $(F(2,344)=49.22, p<.001)$ with perceived stress as the dependent variable, grandiose narcissism showed a weak negative relationship $(\beta=-.15, p<.001)$, while vulnerable narcissism showed a strong positive relationship $(\beta=.44, p<.001)$. Interestingly, these two various forms of narcissism did not relate to each other $(r=.04)$.

In conclusion, we ran a complete regression model with perceived stress regressed on all Dark Triad traits. The result showed that vulnerable narcissism was the one important factor that explained increase in perceived stress ( $\beta=.45, p<.001)$, while grandiose $(\beta=-.16, p<.001)$ and psychopathy $(\beta=-.11, p<.01)$ only showed weak negative relationships. Machiavellianism showed a non-significant effect when controlling for all study variables $(\beta=.04)$.

\section{Discussion}

The main finding in the current study was a strong positive relationship between narcissism and perceived stress, but only for the vulnerable form of narcissism. Grandiose narcissism in contrast had a negative relationship with perceived stress, however weak. This result implies that a vulnerable narcissistic personality disposition relates to increased affect from stressful circumstances, while a grandiose personality in contrast may even thrive in stressful environments. The results, using a general perception stress-scale, imply that there is a significant difference between the two forms of narcissism, which is a confirmation of previous research (Birkás et al. 2016b). The second finding was that psychopathy and Machiavellianism did not relate much with perceived stress, even though psychopathy showed a weak negative relationship. The expected similarity between psychopathy and Machiavellianism was thus only found to a degree. One conclusion is that the Dark Triad is too broad to be seen as one unified construct, and effectively should be applied in its original components.

\section{Malevolent Personality and Stress}

With help of the present perceived stress instrument, we could detect how certain malevolent personality traits relate to everyday life pressures. Most previous studies on the subject have investigated stress behaviors, and not so much latent personality traits. Experiences of stress are most often (if not always) triggered by personal perceptions of situation. We argue that by studying personality traits we move closer to the origins and latent vulnerabilities of stress, which in turn better can predict long-term behaviors, such as health or work performances.

Our present study results showed negative relationships between psychopathy/grandiose narcissism and perceived stress, which aligns with previous research reporting that these are related to lower neuroticism (Gore and Widiger 2016). It is likely that features of the Dark Triad, such as lack of empathy and high self-esteem, buffer against stress. Simply put, if the individual does not care, why would he or she feel stressed? Furthermore, the results for vulnerable narcissism were along expected lines. Speculatively, the main reason for the differences between the two forms of narcissism (vulnerable vs grandiose) in relation to stress may simply be due to differences in levels of underlying neuroticism - Vulnerable narcissism contains much neuroticism, while grandiose even the opposite which can be thought of as resilience (see Miller et al. 2011). The drives behind these can be understood through the lens of Reinforcement Theory, and particularly avoidancemotivation (BIS; Foster and Trimm 2008; Jonason and Jackson 2016). Vulnerable narcissists, sometimes called hyper-sensitive, have a higher avoidance-motivation, while grandiose narcissists have a higher approach-motivation, thus translating into differing stress-levels in the present study. Our contribution to this body of knowledge is that many psychology studies relating to the Dark Triad or everyday perceptions of stress have not treated narcissism in two separate forms, which they according to our study results should (Noser et al. 2014; Richardson and Boag 2016; Paulhus and Williams 2002). Due to the known links between stress and the trait Neuroticism, we believe the present results should be interpreted that persons with vulnerable narcissistic traits more readily perceive situations as threatening to self and thus stressful, rather than seeking out a lifestyle that is more stressful.

Overall, stress is known to have a major impact on people's lives, not least because of long-term sicknesses and decreasing performances (Cohen et al. 1993; Richardson et al. 2012). One implication from the present study is the importance of matching the right person traits to the right job positions; in order to benefit both the employee and the organization as much as possible. A commonly used method of attempting to tackle this issue is different types of personality assessments (Furnham 2008). Vulnerable narcissism may not be much tested and could be suggested as a complement to personality tests in recruitment to particularly stressful services. Dispositions to vulnerability together with being narcissistic may be toxic ingredients for increased perceptions of stress. 


\section{Limitations}

One weakness of the present study was that we only had access to self-reports, which is only one of many ways to assess traits and stress. First, self-selection bias to the voluntary online form may be the case. Second, shared method variance, such as participants rating systematically biased or from a social desirability perspective, may be a threat to validity. However, self-reports are also a strength, especially in attempting to assess perceptions of individuals. Third, several scales showed very low internal consistencies (alpha reliabilities). However, lower reliability does not appear to limit correlations with important external criteria (Miller et al. 2011). Fourth, we mostly had women as participants. Women are known to be somewhat less narcissistic and much less psychopathic than men, and this could have skewed the results (Kajonius et al. 2016). We believe the present study may contribute to stimulating further research in personality traits and everyday life experience.

The present study perhaps couldn't answer the bartender's initial question as to which of the personality types is the darkest (most malevolent), but it can report that vulnerable narcissists experience much more stress than psychopaths, Machiavellians, and grandiose narcissists.

Acknowledgements Thanks to fellow contributor Christina Balder for data collection and being part of the project. Thanks to professor Anna Dåderman for kindly helped reviewing earlier drafts of the manuscript. Thanks to the department of psychology at the University West, Trollhättan, Sweden for making this study possible. On behalf of all authors, the corresponding author states that there is no conflict of interest.

\section{Compliance with Ethical Standards}

Ethical Approval All procedures performed in studies involving human participants were in accordance with the ethical standards of the institutional and/or national research committee and with the 1964 Helsinki declaration and its later amendments or comparable ethical standards. Informed consent was obtained from all individual participants included in the study.

Conflict of Interest Author A declares that he has no conflicts of interest. Author B declares that she has no conflicts of interest.

Open Access This article is distributed under the terms of the Creative Commons Attribution 4.0 International License (http:// creativecommons.org/licenses/by/4.0/), which permits unrestricted use, distribution, and reproduction in any medium, provided you give appropriate credit to the original author(s) and the source, provide a link to the Creative Commons license, and indicate if changes were made.

\section{References}

Birkás, B., Gács, B., \& Csathó, Á. (2016a). Keep calm and don't worry: Different dark triad traits predict distinct coping preferences. Personality and Individual Differences, 88, 134-138. https://doi. org/10.1016/j.paid.2015.09.007.
Birkás, B., Láng, A., Martin, L., \& Kállai, J. (2016b). Disturbing concerns for dark personalities: Anxiety sensitivity and the dark triad. International Journal of Advances in Psychology, 5, 1-5.

Bolger, N., \& Zuckerman, A. (1995). A framework for studying personality in the stress process. Journal of Personality and Social Psychology, 69(5), 890-902. https://doi.org/10.1037/ 0022-3514.69.5.890.

Christie, R., \& Geis, F. L. (1970). Studies in Machiavellianism. New York: Academic Press.

Cohen, S., Kamarck, T., \& Mermelstein, R. (1983). A global measure of perceived stress. Journal of Health and Social Behavior, 24(4), 385-396.

Cohen, S., Tyrrell, D. A. J., \& Smith, A. P. (1993). Negative life events, perceived stress, negative affect, and susceptibility to the common cold. Journal of Personality and Social Psychology, 64(1), 131140. https://doi.org/10.1037/0022-3514.64.1.131.

Collison, K. L., Miller, J. D., Gaughan, E. T., Widiger, T. A., \& Lynam, D. R. (2016). Development and validation of the super-short form of the elemental psychopathy assessment. Journal of Criminal Justice, 47, 143-150. https://doi.org/10. 1016/j.jcrimjus.2016.09.002.

Costa, P. T., \& McCrae, R. R. (1985). The NEO personality inventory. Odessa: Psychological Assesment Resources.

Ebstrup, J. F., Eplov, L. F., Pisinger, C., \& Jørgensen, T. (2011). Association between the five factor personality traits and perceived stress: Is the effect mediated by general self-efficacy? Anxiety, Stress \& Coping: An International Journal, 24(4), 407-419. https://doi.org/10.1080/10615806.2010.540012.

Foster, J. D., \& Trimm IV, R. F. (2008). On being eager and uninhibited: Narcissism and approach-avoidance motivation. Personality and Social Psychology Bulletin, 34(7), 1004-1017.

Furnham, A. (2008). HR professionals' beliefs about, and knowledge of, assessment techniques and psychometric tests. International Journal of Selection and Assessment, 16(3), 300-305.

Furnham, A., Richards, S., Rangel, L., \& Jones, D. N. (2014). Measuring malevolence: Quantitative issues surrounding the dark triad of personality. Personality and Individual Differences, 67(14), 114-121. https://doi.org/10.1016/j.paid.2014.02.001.

Gentile, B., Miller, J. D., Hoffman, B. J., Reidy, D. E., Zeichner, A., \& Campbell, W. K. (2013). A test of two brief measures of grandiose narcissism: The narcissistic personality inventory-13 and the narcissistic personality Inventory-16. Psychological Assessment, 25(4), 1120-1136. https://doi.org/10.1037/a0033192.

Gignac, G. E., \& Szodorai, E. T. (2016). Effect size guidelines for individual differences researchers. Personality and Individual Differences, 10, 274-278. https://doi.org/10.1016/j.paid.2016. 06.069 .

Gore, W. L., \& Widiger, T. A. (2016). Fluctuation between grandiose and vulnerable narcissism. Personality Disorders, 7(4), 363-371. https://doi.org/10.1037/per0000181.

Hare, R. D. (1985). Comparison of procedures for the assessment of psychopathy. Journal of Consulting and Clinical Psychology, 53(1), 7-16. https://doi.org/10.1037/0022006X.53.1.7.

Hendin, H. M., \& Cheek, J. M. (1997). Assessing hypersensitive narcissism: A re-examination of Murray's Narcism scale. Journal of Research in Personality, 31(4), 588-599.

John, O. P., \& Robins, R. (1994). Accuracy and bias in self-perception: Individual differences in self-enhancement and the role of narcissism. Journal of Personality and Social Psychology, 66(1), 206219. https://doi.org/10.1037/0022-3514.66.1.206.

Jonason, P. K., \& Jackson, C. J. (2016). The dark triad traits through the lens of reinforcement sensitivity theory. Personality and Individual Differences, 90, 273-277.

Jones, D. N., \& Paulhus, D. L. (2010). Different provocations trigger aggression in narcissists and psychopaths. Social Psychological 
and Personality Science, 1, 12-18. https://doi.org/10.1177/ 1948550609347591.

Jones, D. N., \& Paulhus, D. L. (2011). The role of impulsivity in the dark triad of personality. Personality and Individual Differences, 51(5), 679-682. https://doi.org/10.1016/j.paid.2011.04.011.

Jones, D. N., \& Paulhus, D. L. (2013). Introducing the short dark triad (SD3): A brief measure of dark personality traits. Assessment, 21(1), 28-41. https://doi.org/10.1177/1073191113514105.

Jones, D. N., \& Weiser, D. A. (2014). Differential infidelity patterns among the dark triad. Personality and Individual Differences, 57, 20-24. https://doi.org/10.1016/j.paid.2013.09.007.

Kajonius, P. J., Persson, B. N., Rosenberg, P., \& Garcia, D. (2016). The (mis) measurement of the dark triad dirty dozen: Exploitation at the core of the scale. PeerJ, 4, e1748.

Klimstra, T. A., Sijtsema, J. J., Henrichs, J., \& Cima, M. (2014). The dark triad of personality in adolescence: Psychometric properties of a concise measure and associations with adolescent adjustment from a multi-informant perspective. Journal of Research in Personality, 53, 84-92. https://doi.org/10.1016/j.jrp.2014.09.001.

Lazarus, R. (1993). From psychological stress to the emotions: A history of changing outlooks. Annual Review of Psychology, 44(1), 1-22.

Lee, K., \& Ashton, M. C. (2005). Psychopathy, Machiavellianism, and narcissism in the five-factor model and the HEXACO model of personality structure. Personality and Individual Differences, 38, 1571-1582. https://doi.org/10.1016/j.paid.2004.09.016.

McHoskey, J. W., Worzel, W., \& Szyarto, C. (1998). Machiavellianism and psychopathy. Journal of Personality and Social Psychology, 74(1), 192-210. https://doi.org/10.1037/0022-3514.74.1.192.

Miller, J. D., Dir, A., Gentile, B., Wilson, L., Pryor, L. R., \& Campbell, W. K. (2010). Searching for a vulnerable dark triad: Comparing factor 2 psychopathy, vulnerable narcissism, and borderline personality disorder. Journal of Personality, 78(5), 1529-1564. https://doi. org/10.1111/j.1467-6494.2010.00660.x.

Miller, J. D., Hoffman, B. J., Gaughan, E. T., Gentile, B., Maples, J., \& Keith Campbell, W. (2011). Grandiose and vulnerable narcissism: A nomological network analysis. Journal of Personality, 79(5), 10131042. https://doi.org/10.1111/j.1467-6494.2010.00711.x.

Miller, J. D., Hyatt, C. S., Maples-Keller, J. L., Carter, N. T., \& Lynam, D. R. (2016). Psychopathy and Machiavellianism: A distinction without a difference? Journal of Personality, 85, 439-453. https://doi. org/10.1111/jopy. 12251.
Neria, A. L., Vizcaino, M., \& Jones, D. N. (2016). Approach/avoidance tendencies in dark personalities. Personality and Individual Differences, 101, 264-269.

Noser, A. E., Zeigler-Hill, V., \& Besser, A. (2014). Stress and affective experiences: The importance of dark personality features. Journal of Research in Personality, 53, 158-164. https://doi.org/10.1016/j.jrp. 2014.10.007.

Paulhus, D. L., \& Williams, K. M. (2002). The dark triad of personality: Narcissism, Machiavellianism, and psychopathy. Journal of Research in Personality, 36(6), 556-563. https://doi.org/10.1016/ S0092-6566(02)00505-6.

Persson, B. N., Kajonius, P. J., \& Garcia, D. (2017a). Revisiting the structure of the short dark triad. Assessment, 1073191117701192, 107319111770119. https://doi.org/10.1177/1073191117701192.

Persson, B. N., Kajonius, P. J., \& Garcia, D. (2017b). Testing construct independence in the short dark triad using item response theory. Personality and Individual Differences, 117, 74-80.

Pincus, A. L., Ansell, E. B., Pimentel, C. A., Cain, N. M., Wright, A. G., \& Levy, K. N. (2009). Initial construction and validation of the pathological narcissism inventory. Psychological Assessment, 21, 365-379. https://doi.org/10.1037/a0016530.

Raskin, R., Novacek, J., \& Hogan, R. (1991). Narcissism, self-esteem, and defensive self-enhancement. Journal of Personality, 59(1), 1938. https://doi.org/10.1111/j.1467-6494.1991.tb00766.x.

Richardson, E. N., \& Boag, S. (2016). Offensive defenses: The mind beneath the mask of the dark triad traits. Personality and Individual Differences, 92, 148-152. https://doi.org/10.1016/j.paid. 2015.12.039.

Richardson, S., Shaffer, J., Falzon, L., Krupka, D., Davidson, K., Edmondson, D., \& Edmondson, D. (2012). Meta-analysis of perceived stress and its association with incident coronary heart disease. American Journal of Cardiology, 110(12), 1711-1716. https://doi. org/10.1016/j.amjcard.2012.08.004.

Vazire, S., \& Funder, D. C. (2006). Impulsivity and the self-defeating behavior of narcissists. Personality and Social Psychology Review, 10(2), 154-165. https://doi.org/10.1207/s15327957pspr1002_4.

Wink, P. (1991). Two faces of narcissism. Journal of Personality and Social Psychology, 61(4), 590-597. https://doi.org/10.1037/00223514.61.4.590. 